\title{
Ministry of Health Clinical Practice Guidelines: Dementia
}

Nagaendran K, Chen LH Christopher, Chong MS, Chua Esther Vanessa, Goh CK Shirley, Kua Joshua, Lee Theresa, Marziyana AR, Ng CC David, Ng LL, Seow Dennis, Sitoh YY, Yap LK Philip, Yeo Donald, Yeo Y

Singapore Med J 2013; 54(5): 293-299; http://dx.doi.org/10.11622/smedj.2013112

The name of one of the authors, "Lim Wee Shiong", was omitted from the article. Thus, the author byline should read:

Nagaendran K, Chen LH Christopher, Chong MS, Chua Esther Vanessa, Goh CK Shirley, Kua Joshua, Lee Theresa, Lim WS, Marziyana AR, Ng CC David, Ng LL, Seow Dennis, Sitoh YY, Yap LK Philip, Yeo Donald, Yeo Y 